Research article

https://doi.org/10.26496/bjz.2017.1

\title{
Effects of an experimental resource pulse on the macrofaunal assemblage inhabiting seagrass macrophytodetritus
}

\author{
François Remy ${ }^{1}$, Sylvie Gobert ${ }^{1} \&$ Gilles Lepoint ${ }^{1,{ }^{*}}$ \\ ${ }^{1}$ Laboratory of Oceanology, FOCUS UR, University of Liège, Belgium. \\ *Corresponding author: G.Lepoint@ulg.ac.be
}

\begin{abstract}
Physical disturbances and resource pulses are major structuring drivers of terrestrial and aquatic ecosystems. The accumulations of exported dead leaves from the Neptune grass, Posidonia oceanica (L.) Delile are ephemeral and highly dynamic detrital habitats offering food sources and shelter for vagile macrofauna community. These habitats are frequently subject to wind and storms which can add "new" detrital material to previous accumulations; these can be defined as resource pulses and could potentially impact the associated macrofauna. This study assesses the impact of an experimental resource pulse on the macrofauna associated with exported $P$. oceanica litter accumulations. The experimental design consisted of two pulse treatments (the addition of dead leaves with and without the associated fauna), and two controls (one procedural, and one total control), where the added material was left underwater for 14 days. Invertebrates then present in the sampled detritus were all identified and counted. Our data suggest that the responses of these invertebrates to resource pulses present intermediate characteristics between aquatic and terrestrial ecosystems responses. Inputting a moderate amount of dead $P$. oceanica leaves into experimental mesocosms had a non-negligible impact and rapidly affected the macrofauna community. Specialist detritivores species were boosted while herbivore/detritivore species dramatically decreased. Predators also showed a modest but significant density increase, demonstrating the fast propagation of the pulse response throughout the entire community and through several trophic levels. Strict hypoxia-tolerant species were also only observed in the treated mesocosms, indicating the strong influence of resource pulses on physico-chemical conditions occurring inside litter accumulations.
\end{abstract}

KEYWORDS. Resource pulses, Posidonia oceanica, macrofauna, experimental ecology, macrophytodetritus.

Remy F., Gobert S. \& Lepoint G. 2017. Effects of an experimental resource pulse on the macrofaunal assemblage inhabiting seagrass macrophytodetritus. Belgian Journal of Zoology 147 (1): 1-15. https://doi.org/10.26496/ bjz.2017.1

\section{Introduction}

In terrestrial, estuarine and marine ecology, disturbances are regarded as playing a central structuring role in ecosystems (GILLER 1996; LAKE 2000). Resource pulses have recently been defined as "rare, brief and intense episodes of increased resource availability in space and time" (OSTFELD \& KEESING 2000; YANG et al. 2008). They can take place in many different ecosystems (e.g., massive floods in arid ecosystems or floodplains, dead leaf litter input in mangroves or forests, massive emergence of insects, mass seeding events or storm driven nutrient runoffs). Some pulses are recurrent in time (e.g., terrestrial 
leaf litter input in autumn in decidual temperate forests) and others are purely stochastic in occurrence, duration, and amplitude (HOLT 2008; NOWLIN et al. 2008; LEVI et al. 2015). Pulses can be caused by different factors: (1) climatic or environmental causes, (2) temporal accumulation and release, (3) spatial accumulation and release, (4) outbreak population dynamics (YANG et al. 2008; LEROUX \& LOREAU 2012). Studying these potentially structuring events is an important topic in the perspective of building a better collective understanding of the controversial climate change impact on extreme stormy events in the Southern European seas (MÖLTER et al. 2016).

The endemic and highly productive Neptune grass, Posidonia oceanica L. Delile, 1813, covers between $25.10^{3} \mathrm{~km}^{2}$ and $45.10^{3} \mathrm{~km}^{2}$ of the Mediterranean coastal area, which represents $1-2 \%$ of the Mediterranean Sea (PASQUALINI et al. 1998). Detrital pathway is considered as a very important pathway for the incorporation of $P$. oceanica organic matter into the coastal food webs as a large amount of the foliar primary production (up to $90 \%$ ) can end up in the detrital compartment (MATEO \& ROMERO 1997; Romero et al. 1992; VALENTINE \& HECK 1999). These dead leaves, once exported out of the meadow to unvegetated places (e.g., bare sand patches) form accumulations associated with bacteria, fungi, microalgae, macroalgae, living leaves, uprooted rhizomes, dead organisms and fine sediment ("exported macrophytodetritus accumulations", hereafter EMAs) (ANESIO et al. 2003; BOUDOURESQUE et al. 2006; LEPOINT et al. 2006; LEMKE et al. 2007; MASCART et al. 2015a).

These EMAs can be considered as constantly subject to the influence of pulsed perturbation (storm, wind events) and resource pulses ("new litter" input) affecting both the resource availability and habitat physico-chemical conditions in the litter. EMAs are known to serve as shelter and food source for a variety (80-115 species) of vagile macroinvertebrates (size $>500 \mu \mathrm{m})($ GALLMETZER et al. 2005; DiMECH et al. 2006; LEPOINT et al. 2006; STURARO et al. 2010; REMY 2016) and are comparable to macrophytodetritus habitats found in Mediterranean estuaries, brackish lakes or lagoons (Phragmites accumulation, for example) (MANCINELLI et al. 2005).

Since these invertebrates live, reproduce and feed in EMAs all year long, resource pulses might potentially play a strong structuring role for this vagile macrofauna community.

To our knowledge, this is the first study that experimentally explores the impact of resource pulses on P. oceanica exported macrophytodetritus accumulations and specifically on the associated macrofauna. We aimed to answer the following questions: (1) Can a clear response of the macrofauna to litter pulses be identified in terms of density and/or diversity? (2) Does the presence/absence of the animal fraction of the litter influence the macrofauna's response to a resource pulse? (3) Do pulsed events maintain biodiversity inside EMAs?

\section{Material and methods}

\section{Sampling site}

The in situ experiment was carried out in 2014, from October $7^{\text {th }}$ to October $21^{\text {st }}$ near the STARESO (STAtion de REcherches Sous-marines et Océanographiques) (University of Liège) research station in Calvi Bay $\left(42^{\circ} 35^{\prime} \mathrm{N} ; 8^{\circ} 43^{\prime} \mathrm{E}\right)$ in Corsica. The sampling site was situated at $10 \mathrm{~m}$ depth on a $200 \mathrm{~m}^{2}$ bare coarse sediment patch regularly covered with dead $P$. oceanica litter, just next to the northwestern part of Punta Oscelluccia (Figure 1). During the whole experimentation period, a constant salinity of 38 and temperatures between 22.8 and $23.1^{\circ} \mathrm{C}$ were observed.

\section{Experimental setup}

Since resource pulses occurring in EMAs consist mainly of dead leaf input, two controls and two treatments $(\mathrm{N}=5)$ were designed to assess the impact of "fresh" litter supply on this community. Each mesocosm $(21 \mathrm{~L})$ consisted of a transparent PVC box $(20 \times 30 \times 35 \mathrm{~cm})$ with a $38 \mu$ m nylon mesh to 
exclude predation by fishes, but allowing free water exchange. The first procedural control, hereafter referred as $\mathbf{C},(\mathrm{N}=5)$ and comprised $\mathrm{PVC}$ mesocosms placed on the EMA without addition of dead leaves. To assess the impact of mesocosms on the macrofauna community, a total control, hereafter referred as $\mathbf{T}_{\text {final }},(\mathrm{N}=6)$ was taken at the end of the experiment. This $\mathrm{T}_{\text {final }}$ was a sample of the "natural" community present in the EMA outside of the mesocosms, on a $20 \times 30 \mathrm{~cm}$ surface, to compare it to the procedural control. The first treatment ( $\mathrm{N}=5$ ), hereafter referred as "T-defaun", was composed of PVC mesocosms containing a given additional supply of "defaunated litter", to assess the potential impact of purely vegetal detrital supply. The second treatment $(\mathrm{N}=5)$, hereafter referred as "T-fauna", was composed of PVC mesocosms each containing a given additional supply of "natural litter", comprising the vegetal detrital material and the associated fauna present inside (fauna was not characterised in this "additional litter", but assumed to be similar to what was identified in the $\mathrm{T}_{\text {final }}$ samples). The litter intended for being added to the two treatments was sampled $24 \mathrm{~h}$ before the beginning of the experiment; half of it was defaunated and the other half was kept in $750 \mathrm{~L}$ storage. Defaunation was achieved by rinsing the
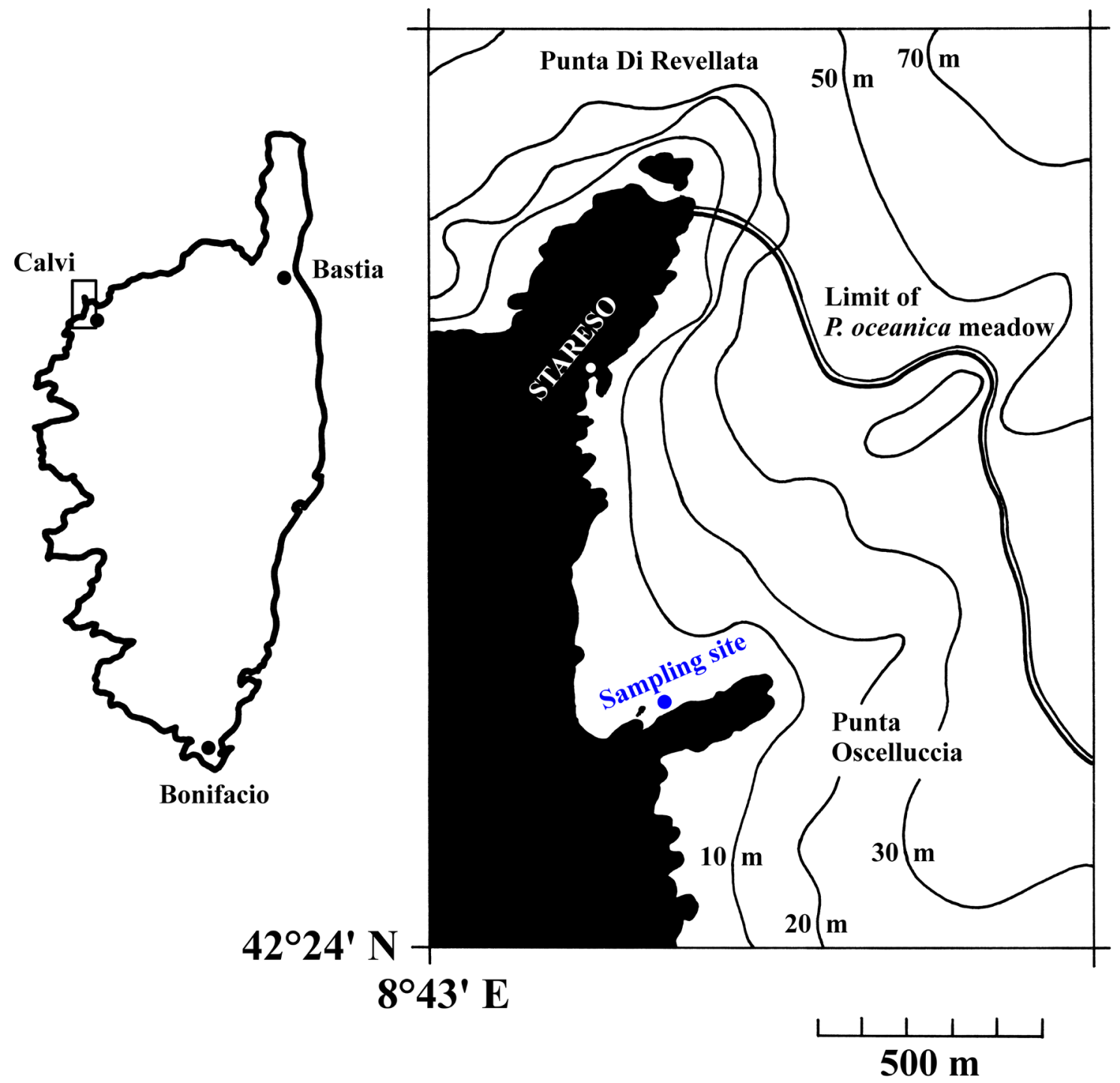

Figure 1 - Location of the STARESO research station (University of Liège) and the sampling site in the Bay of Calvi indicating the lower limit of the P. oceanica meadow (modified after GOBERT 2002). 
sampled litter on a $10 \mathrm{~mm}$ sieve stacked on a $500 \mu \mathrm{m}$ nylon-mesh sieve in order to separate the detrital material from the macrofauna.

The 15 mesocosms were placed at a depth of $9 \mathrm{~m}$ at the sampling site (Figure 1) and on an adjacent EMA situated directly north-east. Treatments and controls were placed randomly to prevent any bias from a potential position effect. Just before starting the experiment, $205 \pm 3 \mathrm{~g}$ (wet mass) of litter were placed inside each mesocosm for both treatments. This amount of litter represented visually a doubling $(+100 \%)$ of the amount of litter naturally present at the sampling site at the beginning of the experiment. This was considered as corresponding to a "moderate" resource pulse in terms of litter input (see Remy 2016). Mesocosms were anchored on the sediment with marine steel poles and weights to prevent any movement of the setup and avoid dead leaf movements in or out of the mesocosm-sediment interface. This anchoring did not prevent the possibility of colonisation/escaping by macrofauna inhabiting the surrounding litter. After 14 days, all mesocosms were sealed underwater and brought back to the lab for further processing.

\section{Sample processing}

In the laboratory, macrofauna was separated from the dead P. oceanica leaves using freshwater on successive $10 \mathrm{~mm}$ and $500 \mu \mathrm{m}$ sieves for optimal and handy separation. The $500 \mu \mathrm{m}$ fraction was preserved in a $4 \%$ formaldehyde seawater solution and kept for further analysis. After $48 \mathrm{~h}$ of formaldehyde fixation, the $4 \%$ formaldehyde seawater solution was replaced by distilled water for final sorting. Specific identification and counting of all individuals was effected using a stereomicroscope (Zeiss Stemi 2000-C). Animals were then stored in $99.8 \%$ ethanol. The remaining defaunated detrital fraction was dried at $60{ }^{\circ} \mathrm{C}$ for 5 days and then weighed. Density data are, therefore, expressed per gram of dried litter present in the mesocosm at the end of the experiment. All presented data are presented in the form of mean \pm standard deviation.

\section{Data analysis}

A non-metric multidimensional scaling (n-MDS) coupled with an ANOSIM analysis was performed on the square root-transformed density data of all the sampled species. The n-MDS analysis is based on an iterative procedure. For a given number of dimensions, many iterations are computer-generated. Each iteration corresponds to a possible ordination. A "stress" value is calculated for each attempt, which is in fact a way to express the error associated with the ordination procedure, i.e., the mismatch between theoretical inter-sample similarities and real similarities between those samples, measured on the ordination. The iteration that shows the lowest stress value is then considered as being the best way to map the samples. In this study, we performed a 2D n-MDS using the "MDS" routine of PRIMER v6.1.13 for Windows. The resemblance matrix was built by calculating Bray-Curtis similarity. The number of iterations was set to 99 , and the minimum stress level at 0.01 . ANOSIM analysis is widely used in ecology and has some analogies with ANOVA-like analysis; however, it is used to evaluate directly a dissimilarity matrix rather than raw data. Together with the complementary n-MDS, this analysis is adapted to differentiate two or more groups for multivariate data.

A SIMPER analysis was also performed. The purpose of one-way SIMPER (SIMilarity PERcentage) analyses is to highlight the variables (here, species) best explaining the similarity between samples forming a single group and dissimilarity between two groups. The program calculates Bray-Curtis similarity between all pairs of items of a group. In parallel to this similarity breakdown procedure, the program also performs an inter-group dissimilarity breakdown. This procedure is very similar to the similarity breakdown described above but is based on the breakdown of the total inter-group dissimilarity, giving the relative contribution (expressed in \%) of each variable (here, species) to the dissimilarity between groups. 
The widely used Shannon-Wiener index (hereafter, H') and Simpson index (hereafter, 1- $\lambda$ ') were also calculated for each sample.

Classical statistical analysis (ANOVA and MANOVA) were performed using R. nMDS, ANOSIM and SIMPER analyses were performed using PRIMER 6.1.13 (CLARKE \& GORLEY 2006) with PERMANOVA additional software (ANDERSON et al. 2008b). A significance level of $\mathrm{p}<0.01$ was used in all tests to limit "Type I error".

Graphs were built with PRIMER 6.1.13 and GraphPad PRISM 6.01 software for Windows (GraphPad Software, San Diego, USA).

\section{Results}

At the end of the experiment, there was no significant difference in litter biomass sampled inside the mesocosms composing the four treatments (1-way ANOVA, $\mathrm{p}=0.5024$ ).

A total of 3503 individuals from 36 species were sampled at the end of the 14 days in situ experiment, representing a mean global density of $4.4 \pm 1.4$ ind. $\mathrm{gDM}^{-1}$. A total of 25 species were sampled in the total control $\left(\mathbf{T}_{\text {final }}\right), 26$ species in the control (hereafter referred as "C"), 35 species in the defaunated litter treatment (T-defaun.) and 36 species in the litter + fauna treatment (T-fauna).

Arthropods (24 species) were extremely dominant, representing on average $85.6 \pm 2.8 \%(3.54 \pm 1.2$ ind. $\mathrm{gDM}^{-1}$ ) of the whole sampled community (Table 1 ). Within arthropods, amphipods were the most dominant taxa, representing $78.2 \pm 12.5 \%$ of arthropod abundance, followed by decapods representing $9.6 \pm 6.1 \%$, isopods representing $7.3 \pm 3.3 \%$ and leptostraceans representing $4.7 \pm 3.8 \%$ (Table 1 ). The amphipod Gammarella fucicola (Leach, 1814) was typically the most abundant species, representing by itself $62.9 \pm 17.6 \%$ of the amphipods and $44.0 \pm 20.2 \%\left(1.9 \pm 1.3\right.$ ind. $\left.\mathrm{gDM}^{-1}\right)$ of the total macrofauna. Arthropods were followed by annelids (6 species) representing $8.2 \pm 2.2 \%\left(0.4 \pm 0.2\right.$ ind. $\left.\mathrm{gDM}^{-1}\right)$, mollusks (4 species) representing $4.2 \pm 1.7 \%\left(0.2 \pm 0.1\right.$ ind. $\left.\mathrm{gDM}^{-1}\right)$ and echinoderms $(2$ species $)$ representing $2.03 \pm 1.33 \%\left(0.1 \pm 0.04\right.$ ind. $\left.\mathrm{gDM}^{-1}\right)$ of the community.

A preliminary MANOVA (1-way MANOVA, $p>0.95)$ demonstrated that the total control $\left(\mathrm{T}_{\text {final }}\right)$ was not different from the procedural control (C) for either global density, $\mathrm{H}^{\prime}, 1-\lambda$ ' or for diversity at high level taxa and at specific level. Since the "mesocosm effect" was negligible, and in order to simplify future analyses, $\mathrm{T}_{\text {final }}$ was not included any further in the next MANOVAs. Another MANOVA analysis showed that global density and high taxonomic level data presented no significant differences (1way MANOVA, $p>0.06$ ) according to the treatment. On the other hand, density data of amphipods, decapods and leptostraceans presented highly significant differences according to the treatment (1-way MANOVA, $\mathrm{p}<0.0005$ and ANOVA, $\mathrm{p}<0.0002$ ).

The n-MDS analysis based on the squared density data of the 36 species showed clearly this pattern, forming two main significant (ANOSIM, $\mathrm{p}<0.008$ ) groups; one of them being the "Control group" composed of $\mathrm{C}$ replicates and $\mathrm{T}_{\text {final }}$ replicates, and the significantly different other group being the "Pulse cluster" composed of T-defaun. and T-fauna replicates (Figure 2).

The Shannon-Wiener diversity index (H') and Simpson evenness index (1- $\left.\lambda^{\prime}\right)$ also showed important variations from one treatment to the other. $\mathrm{H}^{\prime}$ index showed a minimum value of $2.42 \pm 0.17 \mathrm{in} \mathrm{C}$, and values of $2.94 \pm 0.16$ and $3.01 \pm 0.15$ in T-defaun. and T-fauna respectively, indicating a lower diversity in C. $1-\lambda$ ' index showed a minimum value of $0.86 \pm 0.03$ in $C$, and a maximum value of $0.94 \pm 0.01$ in both T-defaun. and T-fauna, indicating that a few species were highly dominant in all treatments and that their dominance increased in T-defaun. and T-fauna.

Multivariate analysis (1-way MANOVA, $\mathrm{p}<0.0001$ and ANOVA, $\mathrm{p}<0.0001$ ) showed that both H' and $1-\lambda$ ' were significantly influenced by the treatment. 


\section{TABLE 1}

Density of macrofauna found in experimental treatment. Results are mean \pm standard deviation.

\begin{tabular}{|c|c|c|c|c|c|c|c|c|c|c|c|c|}
\hline & \multicolumn{3}{|c|}{$\mathrm{T}_{\text {final }}$} & \multicolumn{3}{|c|}{ Control } & \multicolumn{3}{|c|}{ T-defaun } & \multicolumn{3}{|c|}{ T-fauna } \\
\hline & Avg. & \pm & SD & Avg. & \pm & SD & Avg. & \pm & SD & Avg. & \pm & SD \\
\hline Gammarella fucicola & 3.28 & \pm & 0.51 & 3.5 & \pm & 1 & 1.14 & \pm & 0.19 & 1.08 & \pm & 0.19 \\
\hline Gammarus aequicauda & 0.13 & \pm & 0.04 & 0.11 & \pm & 0.06 & 0.7 & \pm & 0.18 & 0.72 & \pm & 0.12 \\
\hline Melita hergensis & 0.17 & \pm & 0.12 & 0.19 & \pm & 0.16 & 0.13 & \pm & 0.08 & 0.11 & \pm & 0.04 \\
\hline Apherusa chiereghinii & 0.03 & \pm & 0.04 & 0.01 & \pm & 0.02 & 0.01 & \pm & 0.01 & 0.03 & \pm & 0.02 \\
\hline Leptocheirus guttatus & 0.01 & \pm & 0.01 & 0.01 & \pm & 0.01 & 0.01 & \pm & 0.02 & 0.02 & \pm & 0.03 \\
\hline Microdeutopus chelifer & 0.21 & \pm & 0.11 & 0.25 & \pm & 0.2 & 0.11 & \pm & 0.06 & 0.04 & \pm & 0.03 \\
\hline Stenothoe monoculoides & 0.01 & \pm & 0.02 & 0.02 & \pm & 0.03 & 0.01 & \pm & 0.01 & 0.01 & \pm & 0.01 \\
\hline Lysianassa costae & & & & & & & 0.02 & \pm & 0.03 & 0.02 & \pm & 0.03 \\
\hline Nototropis guttatus & 0.05 & \pm & 0.03 & 0.06 & \pm & 0.04 & 0.03 & \pm & 0.02 & 0.09 & \pm & 0.04 \\
\hline Maera grossimana & 0.01 & \pm & 0.01 & 0.01 & \pm & 0.01 & 0.01 & \pm & 0.02 & 0.01 & \pm & 0.01 \\
\hline Dexamine spinosa & 0.03 & \pm & 0.03 & 0.02 & \pm & 0.02 & 0.01 & \pm & 0.02 & 0.02 & \pm & 0.03 \\
\hline Athanas nitescens & 0.02 & \pm & 0.03 & 0.03 & \pm & 0.04 & 0.13 & \pm & 0.02 & 0.15 & \pm & 0.06 \\
\hline Palaemon xiphias & 0.04 & \pm & 0.02 & 0.05 & \pm & 0.04 & 0.08 & \pm & 0.01 & 0.18 & \pm & 0.07 \\
\hline Alpheus glaber & 0.01 & \pm & 0.01 & 0 & \pm & 0.01 & 0.02 & \pm & 0.05 & 0.03 & \pm & 0.02 \\
\hline Galathea intermedia & 0.02 & \pm & 0.03 & 0.01 & \pm & 0.02 & 0.01 & \pm & 0.02 & 0.07 & \pm & 0.03 \\
\hline Anapagurus chiroacanthus & & & & & & & 0.07 & \pm & 0.06 & 0.07 & \pm & 0.03 \\
\hline Pisa tetraodon & 0.01 & \pm & 0.01 & 0.01 & \pm & 0.01 & & & & 0.01 & \pm & 0.01 \\
\hline Liocarcinus navigator & 0.02 & \pm & 0.03 & 0.02 & \pm & 0.04 & 0.01 & \pm & 0.01 & 0.01 & \pm & 0.02 \\
\hline Jaera nordmanni & 0.13 & \pm & 0.09 & 0.15 & \pm & 0.12 & 0.2 & \pm & 0.05 & 0.24 & \pm & 0.04 \\
\hline Idotea balthica & & & & & & & 0.02 & \pm & 0.02 & 0.02 & \pm & 0.03 \\
\hline Stenosoma lancifer & 0.02 & \pm & 0.03 & 0.02 & \pm & 0.04 & 0.03 & \pm & 0.04 & 0.01 & \pm & 0.02 \\
\hline Cymodoce truncata & & & & & & & 0.02 & \pm & 0.01 & 0.02 & \pm & 0.02 \\
\hline Nebalia strausi & & & & & & & 0.19 & \pm & 0.08 & 0.24 & \pm & 0.06 \\
\hline Achelia echinata & & & & 0.01 & \pm & 0.01 & 0.01 & \pm & 0.02 & 0.01 & \pm & 0.01 \\
\hline Platynereis dumerilii & 0.24 & \pm & 0.13 & 0.3 & \pm & 0.21 & 0.08 & \pm & 0.04 & 0.15 & \pm & 0.03 \\
\hline Polyophthalmus pictus & 0.16 & \pm & 0.04 & 0.15 & \pm & 0.04 & 0.08 & \pm & 0.05 & 0.08 & \pm & 0.05 \\
\hline Hesiospina autantiaca & & & & & & & 0.03 & \pm & 0.02 & 0.04 & \pm & 0.03 \\
\hline Chrysopetalum debile & & & & & & & 0.03 & \pm & 0.02 & 0.05 & \pm & 0.04 \\
\hline Protodorvillea kefersteini & & & & & & & 0.02 & \pm & 0.02 & 0.02 & \pm & 0.02 \\
\hline Hesione panthernia & & & & & & & 0.01 & \pm & 0.01 & 0.01 & \pm & 0.01 \\
\hline Bittium reticulatum & 0.15 & \pm & 0.1 & 0.17 & \pm & 0.14 & 0.11 & \pm & 0.05 & 0.12 & \pm & 0.06 \\
\hline Tricolia tenuis & 0.02 & \pm & 0.03 & 0.03 & \pm & 0.04 & 0.03 & \pm & 0.03 & 0.03 & \pm & 0.04 \\
\hline Rissoa violacea & 0.01 & \pm & 0.02 & 0.01 & \pm & 0.02 & 0.01 & \pm & 0.02 & 0.01 & \pm & 0.01 \\
\hline Chiton olivaceus & & & & & & & 0.01 & \pm & 0.01 & 0.01 & \pm & 0.01 \\
\hline Ampipholis squamata & 0.03 & \pm & 0.03 & 0.02 & \pm & 0.02 & 0.08 & \pm & 0.05 & 0.09 & \pm & 0.03 \\
\hline Holothuria sp. & 0.01 & \pm & 0.02 & 0.01 & \pm & 0.02 & 0.01 & \pm & 0.02 & 0.01 & \pm & 0.01 \\
\hline
\end{tabular}


Fifteen species made up to $90 \%$ of all sampled individuals and only those 15 species will be discussed in detail. However, although representing a relatively limited contribution, the shrimp Palaemon xiphias (Risso, 1816), the only big predator found in EMAs was added to the analysis to monitor the potential impact of a pulsed event on a predator species. Multivariate analysis based on the density of these 16 species showed a significant effect of the treatment on population densities for only 7 of them (1-way MANOVA, p < 0.001). Two amphipod species ( . fucicola and G. aequicauda) and the leptostracean species (Nebalia strausi (Risso, 1826)) showed very highly significant effects of the treatment on population densities (ANOVA, $\mathrm{p}<0.00001$ ). The three decapod species (Athanas nitescens (Leach, 1813), P. xiphias and Galathea intermedia (Lilljeborg, 1851)) showed significant effects of the treatment on population densities (ANOVA, $\mathrm{p}<0.01$ ). The remaining amphipod species (Nototropis guttatus (Costa, 1853)) showed no significant effect on population density. For these 7 species, densities show drastic variations between $\mathrm{C}$, on the one hand, and the pulse treatments on the other (Figure 3). Gammarella fucicola showed a much higher density in C than in T-defaun. or T-fauna. The 6 other species showed an opposite pattern with lower densities in C. Gammarus aequicauda experienced by far the most drastic differences, going from a density of $0.11 \pm 0.06$ ind. $\mathrm{gDM}^{-1}$ in $\mathrm{C}$, to $0.71 \pm 0.13$ ind. $\mathrm{gDM}^{-1}$ in the two pulsed treatments. Decapods and the leptostracean $N$. strausii showed similar trends as in G. aequicauda.

SIMPER similarity analysis for treatment factor based on density data for the 36 species showed that G. fucicola was always the strongest contributor to similarity (Table 2). Similarity was high for all

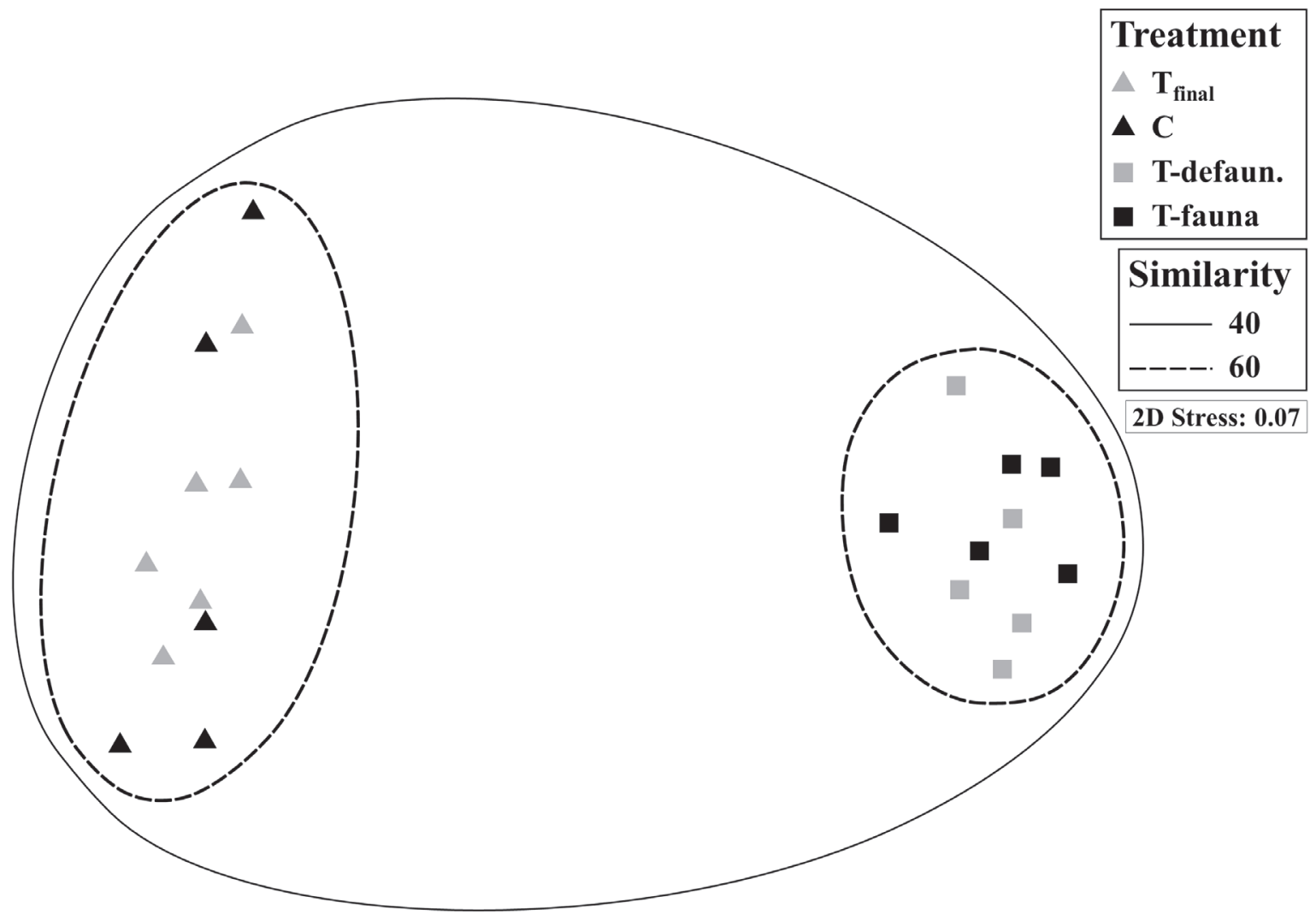

Figure 2-2D n-MDS ordination of the 21 samples and superposed Bray-Curtis similarity curves based on the square root-transformed density data of the 36 sampled species. Similarity curves represent $40 \%$ (solid black) and 60\% (dotted black) of similarity between samples. Black and grey triangles represent $\mathrm{C}$ and $\mathrm{T}_{\text {final }}$, respectively; and black and grey squares represent $\mathrm{T}$-fauna and T-defaun., respectively. 
TABLE 2

Summary table of the SIMPER analysis with factor "treatment" for macrofauna species contribution to intra-group similarity, and inter-group dissimilarity.

\begin{tabular}{|c|c|c|c|c|c|}
\hline \multicolumn{6}{|l|}{ Treatment factor } \\
\hline \multicolumn{3}{|c|}{$\begin{array}{c}\mathrm{T}_{\text {final }} \\
\text { (76.91\% similarity) }\end{array}$} & \multicolumn{3}{|c|}{$\begin{array}{c}\text { Control } \\
\text { (71.35\% similarity) }\end{array}$} \\
\hline Species & $\%$ & $\begin{array}{c}\% \\
\text { Cum. }\end{array}$ & Species & $\%$ & $\begin{array}{c}\% \\
\text { Cum. }\end{array}$ \\
\hline Gammarella fucicola & 37.07 & 37.07 & Gammarella fucicola & 38.54 & 38.54 \\
\hline Platynereis dumerilii & 8.53 & 45.60 & Platynereis dumerilii & 9.25 & 47.79 \\
\hline Polyophthalmus pictus & 7.70 & 53.30 & Polyophthalmus pictus & 8.09 & 55.88 \\
\hline Microdeutopus chelifer & 7.15 & 60.45 & Jaera nordmanni & 7.07 & 62.95 \\
\hline Melita hergensis & 6.35 & 66.80 & Bittium reticulatum & 6.44 & 69.39 \\
\hline Bittium reticulatum & 6.19 & 72.99 & Microdeutopus chelifer & 6.39 & 75.77 \\
\hline Jaera nordmanni & 6.17 & 79.16 & Gammarus aequicauda & 6.21 & 81.98 \\
\hline Gammarus aequicauda & 5.90 & 85.06 & Melita hergensis & 5.46 & 87.44 \\
\hline Nototropis guttatus & 3.53 & 88.59 & Nototropis guttatus & 3.78 & 91.23 \\
\hline Palaemon xiphias & 3.51 & 92.09 & & & \\
\hline \multicolumn{3}{|c|}{ T-defaun. $(75.99 \%$ similarity $)$} & \multicolumn{3}{|c|}{ T-fauna ( $78.56 \%$ similarity) } \\
\hline Species & $\%$ & $\begin{array}{c}\% \\
\text { Cum. }\end{array}$ & Species & $\%$ & $\begin{array}{c}\% \\
\text { Cum. }\end{array}$ \\
\hline Gammarella fucicola & 18.19 & 18.19 & Gammarella fucicola & 15.54 & 15.54 \\
\hline Gammarus aequicauda & 14.02 & 32.21 & Gammarus aequicauda & 12.66 & 28.20 \\
\hline Jaera nordmanni & 7.50 & 39.71 & Jaera nordmanni & 7.34 & 35.54 \\
\hline Nebalia strausi & 6.71 & 46.42 & Nebalia strausi & 7.20 & 42.73 \\
\hline Athanas nitescens & 6.12 & 52.54 & Palaemon xiphias & 5.71 & 48.44 \\
\hline Bittium reticulatum & 4.99 & 57.53 & Platynereis dumerilii & 5.64 & 54.08 \\
\hline Melita hergensis & 4.95 & 62.48 & Athanas nitescens & 5.24 & 59.32 \\
\hline Palaemon xiphias & 4.91 & 67.39 & Melita hergensis & 4.64 & 63.96 \\
\hline Microdeutopus chelifer & 4.60 & 71.99 & Bittium reticulatum & 4.47 & 68.43 \\
\hline Platynereis dumerilii & 4.12 & 76.11 & Ampipholis squamata & 4.17 & 72.60 \\
\hline Polyophthalmus pictus & 3.70 & 79.81 & Nototropis guttatus & 4.07 & 76.67 \\
\hline Ampipholis squamata & 3.36 & 83.17 & Anapagurus chiroacanthus & 3.64 & 80.31 \\
\hline Anapagurus chiroacanthus & 3.22 & 86.39 & Galathea intermedia & 3.40 & 83.71 \\
\hline Hesiospina autantiaca & 2.05 & 88.44 & Polyophthalmus pictus & 3.25 & 86.96 \\
\hline \multirow[t]{2}{*}{ Nototropis guttatus } & 1.79 & 90.23 & Hesiospina autantiaca & 2.56 & 89.52 \\
\hline & & & Chrysopetalum debile & 1.92 & 91.44 \\
\hline
\end{tabular}

\begin{tabular}{|c|c|c|c|c|c|}
\hline \multicolumn{6}{|c|}{ Dissimilarity } \\
\hline \multicolumn{3}{|c|}{$\begin{array}{c}\mathrm{T}_{\text {final }} \text { \& Control } \\
\text { (22.39\% dissimilarity) }\end{array}$} & \multicolumn{3}{|c|}{$\begin{array}{c}\mathrm{T}_{\text {final }} \& \text { T-defaun. } \\
\text { (41.32\% dissimilarity) }\end{array}$} \\
\hline Species & $\%$ & $\begin{array}{c}\% \\
\text { Cum. }\end{array}$ & Species & $\%$ & $\begin{array}{c}\% \\
\text { Cum. }\end{array}$ \\
\hline Gammarella fucicola & 8.14 & 8.14 & Gammarella fucicola & 14.11 & 14.11 \\
\hline Microdeutopus chelifer & 7.14 & 15.27 & Gammarus aequicauda & 9.16 & 23.27 \\
\hline Melita hergensis & 6.12 & 21.39 & Nebalia strausi & 7.69 & 30.96 \\
\hline Platynereis dumerilii & 5.50 & 26.89 & Athanas nitescens & 4.62 & 35.57 \\
\hline Bittium reticulatum & 4.76 & 31.65 & Anapagurus chiroacanthus & 4.35 & 39.92 \\
\hline Tricolia tenuis & 4.63 & 36.28 & Platynereis dumerilii & 3.56 & 43.48 \\
\hline Athanas nitescens & 4.61 & 40.89 & Ampipholis squamata & 3.19 & 46.67 \\
\hline Apherusa chiereghinii & 4.40 & 45.29 & Microdeutopus chelifer & 3.08 & 49.75 \\
\hline Liocarcinus navigator & 4.28 & 49.57 & Hesiospina autantiaca & 2.92 & 52.67 \\
\hline Jaera nordmanni & 4.14 & 53.71 & Chrysopetalum debile & 2.69 & 55.36 \\
\hline Stenosoma lancifer & 4.14 & 57.84 & Melita hergensis & 2.43 & 57.80 \\
\hline Ampipholis squamata & 4.01 & 61.85 & Jaera nordmanni & 2.40 & 60.20 \\
\hline
\end{tabular}

\begin{tabular}{|c|c|c|c|c|c|}
\hline \multicolumn{3}{|c|}{$\begin{array}{l}\text { Control \& T-defaun. } \\
\text { (42.17\% dissimilarity) }\end{array}$} & \multicolumn{3}{|c|}{$\begin{array}{c}\mathrm{T}_{\text {final }} \& \mathrm{~T} \text {-fauna } \\
\text { (41.31\% dissimilarity) }\end{array}$} \\
\hline Species & $\%$ & $\begin{array}{c}\% \\
\text { Cum. }\end{array}$ & ies & $\%$ & $\begin{array}{c}\% \\
\text { Cum } \\
\end{array}$ \\
\hline Gammarella fucicola & 13.63 & 13.63 & Gammarella fucicola & 13.83 & 13.83 \\
\hline Gammarus aequicauda & 9.32 & 22.95 & Gammarus aequicauda & 8.89 & 22.72 \\
\hline Nebalia strausi & 7.60 & 30.55 & Nebalia strausi & 8.34 & 31.06 \\
\hline Anapagurus chiroacanthus & 4.29 & 34.84 & Athanas nitescens & 4.75 & 35.82 \\
\hline Athanas nitescens & 4.16 & 39.00 & Microdeutopus chelifer & 4.53 & 40.35 \\
\hline Platynereis dumerilii & 4.11 & 43.11 & Anapagurus chiroacanthus & 4.41 & 44.75 \\
\hline Microdeutopus chelifer & 3.52 & 46.63 & Palaemon xiphias & 3.62 & 48.37 \\
\hline Ampipholis squamata & 3.42 & 50.05 & Hesiospina autantiaca & 3.41 & 51.79 \\
\hline Hesiospina autantiaca & 2.88 & 52.93 & Chrysopetalum debile & 3.26 & 55.05 \\
\hline Melita hergensis & 2.88 & 55.81 & Galathea intermedia & 2.80 & 57.85 \\
\hline Jaera nordmanni & 2.69 & 58.50 & Ampipholis squamata & 2.78 & 60.63 \\
\hline
\end{tabular}

\begin{tabular}{|c|c|c|c|c|c|}
\hline \multicolumn{3}{|c|}{$\begin{array}{c}\text { Control \& T-fauna } \\
(42.68 \% \text { dissimilarity })\end{array}$} & \multicolumn{3}{|c|}{$\begin{array}{l}\text { T-defaun. \& T-fauna } \\
(22.93 \% \text { dissimilarity })\end{array}$} \\
\hline Species & $\%$ & $\begin{array}{c}\% \\
\text { Cum. }\end{array}$ & Species & $\%$ & $\begin{array}{c}\% \\
\text { Cum. }\end{array}$ \\
\hline Gammarella fucicola & 13.2 & 13.2 & Galathea intermedia & 5.28 & 5.28 \\
\hline Gammarus aequicauda & 8.93 & 22.13 & Microdeutopus chelifer & 4.40 & 9.68 \\
\hline Nebalia strausi & 8.13 & 30.27 & Nototropis guttatus & 4.25 & 13.92 \\
\hline Microdeutopus chelifer & 4.66 & 34.92 & Alpheus glaber & 4.20 & 18.13 \\
\hline Anapagurus chiroacanthus & 4.30 & 39.22 & Palaemon xiphias & 3.78 & 21.91 \\
\hline Athanas nitescens & 4.28 & 43.5 & Tricolia tenuis & 3.52 & 25.43 \\
\hline Palaemon xiphias & 3.73 & 47.23 & Apherusa chiereghinii & 3.50 & 28.94 \\
\hline Hesiospina autantiaca & 3.33 & 50.56 & Lysianassa costae & 3.41 & 32.34 \\
\hline Chrysopetalum debile & 3.18 & 53.74 & Platynereis dumerilii & 3.24 & 35.58 \\
\hline Galathea intermedia & 3.09 & 56.83 & Stenosoma lancifer & 3.21 & 38.79 \\
\hline Ampipholis squamata & 3.08 & 59.9 & Chrysopetalum debile & 3.18 & 41.96 \\
\hline \multirow[t]{6}{*}{ Jaera nordmanni } & 2.93 & 62.83 & Cymodoce truncata & 3.11 & 45.08 \\
\hline & & & Idotea baltica basteri & 3.03 & 48.11 \\
\hline & & & Polyophthalmus pictus & 2.93 & 51.04 \\
\hline & & & Gammarus aequicauda & 2.83 & 53.87 \\
\hline & & & Gammarella fucicola & 2.79 & 56.66 \\
\hline & & & $\begin{array}{l}\text { Leptocheirus guttatus } \\
\text { Dexamine spinosa }\end{array}$ & $\begin{array}{l}2.77 \\
2.73\end{array}$ & $\begin{array}{l}59.44 \\
62.17\end{array}$ \\
\hline
\end{tabular}


treatments ranging from 71.4 to $78.6 \%$. Specific contributions showed a similar pattern in $\mathrm{C}$ and in $\mathrm{T}_{\text {final }}$ and this pattern was very different from the one observed in T-defaun. or T-fauna treatments. An important result was that in $\mathrm{C}$ or $\mathrm{T}_{\text {final }}$, G. fucicola was by far and away the species contributing most to the similarity, while in T-defaun. and T-fauna, G. fucicola was followed closely by G. aequicauda. SIMPER dissimilarity analysis showed that dissimilarity between both treatments and $\mathrm{C}$ or $\mathrm{T}_{\text {final }}$ was much higher (Table 2) than any dissimilarity between the treatments. It also showed that the species explaining most of the dissimilarity between the treatments and the controls were G. fucicola, G. aequicauda and $N$. strausi, which is consistent with what was observed in terms of density variations.

\section{Discussion}

This in situ experiment demonstrated the potential impact of resource pulses on the macrofauna associated to exported dead litter from P. oceanica, an ephemeral habitat characteristic of the Mediterranean coastal zone.

The global density, diversity and dominance patterns observed in the control during this experiment correspond to previous studies of this coastal detrital community (GALLMETZER et al. 2005; DIMECH et al. 2006; REMY 2016), showing lower diversity but higher abundance than in the adjacent seagrass meadow (StURARO et al. 2014; Michel et al. 2015) and higher diversity and higher abundance than in reed macrophytodetritus from Mediterranean brackish water (MANCINELLI et al. 2007) or seagrass (Cymodocea nodosa/Zostera spp.) detritus from the Po estuary (MANCINELli \& Rossi 2002).

The two controls showed no significant difference between each other either, demonstrating that the effect of the experimental design was quite negligible compared to the effect of the treatments (i.e., addition of litter).

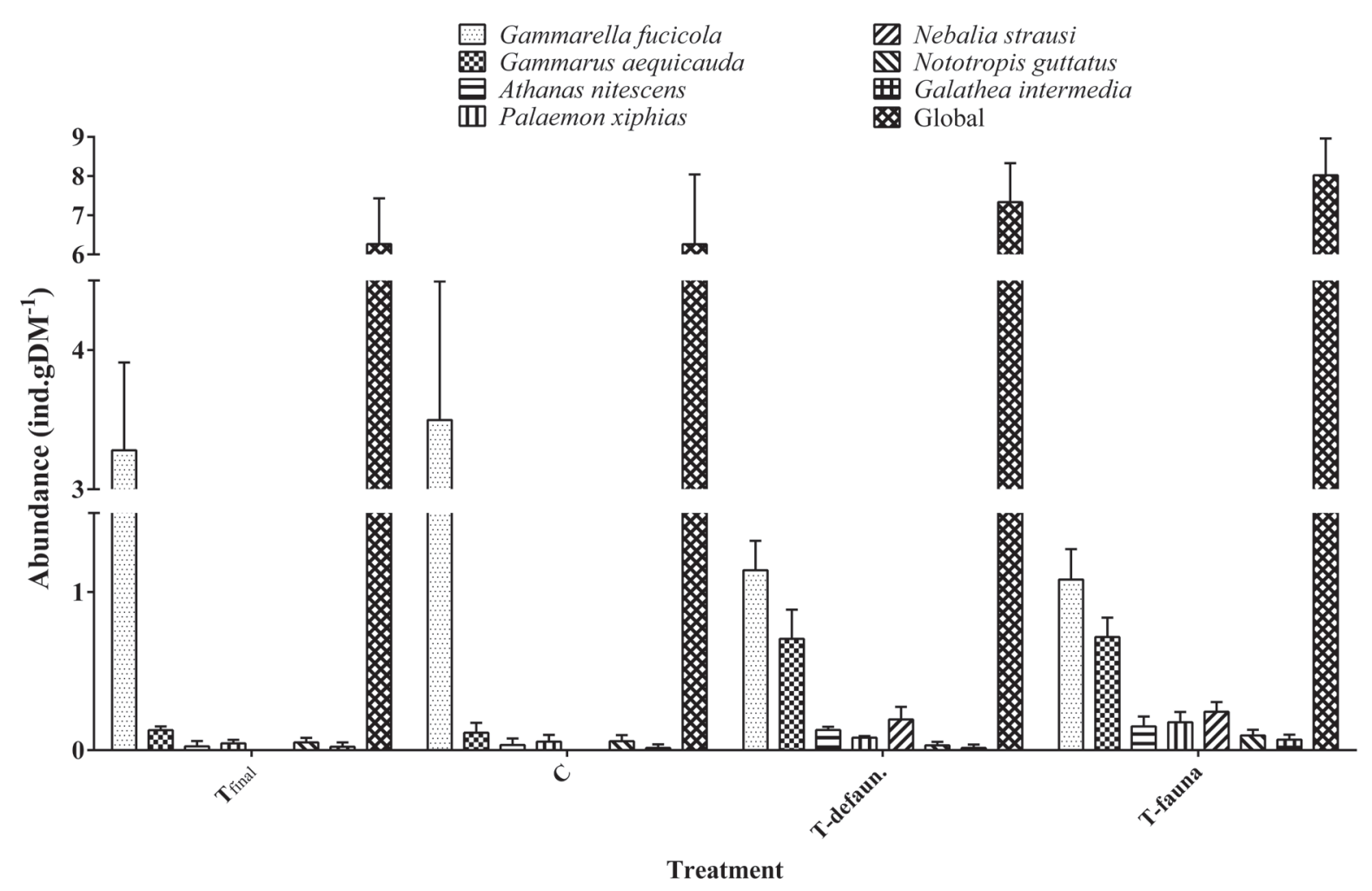

Figure 3 - Mean densities of most abundant species in relation to treatment. Error bars are standard deviations. 
This experiment showed that diversity $\left(H^{\prime}\right)$ and dominance $\left(1-\lambda^{\prime}\right)$ in pulsed litter were influenced by both pulse treatments, and that diversity increased significantly with the addition of dead leaves, with or without the associated macrofauna (diversity: increase of 9-11 species between the control and the treatments). The dominance of some species also significantly increased, indicating that while the number of species encountered increased, a limited number of species seemed literally boosted by the addition of the dead leaves. Such important density variations observed in the relatively short duration of the experiment (14 days) leads to the conclusion that active colonisation and (e)migration of macro-invertebrates have more than likely occurred during the experiment (see MASCART et al. 2015b for meiofauna). Such short term migration/emigration is also observed in reed macrophytodetritus accumulations, occurring over even shorter time scales (i.e., within days) and explaining the non-random variability of macrofauna in these accumulations (MANCINELLI et al. 2005). In our experiment, the mesocosm design allows fauna movement inside the litter or via the sediment. Such behavioural aggregation of consumers is a common fast response to resource pulses (ANDERSON et al. 2008a; HOLT 2008; YANG et al. 2008; YANG et al. 2010; MASCART et al. 2015b) and the linked density variations observed in both treatments could be considered as a community-level 'first response' to a dead leaf pulse.

Another major result was the absence of effect of the addition of the animal fraction on the global abundance of the macrofauna community. Moreover, there were no differences in global abundance between $\mathrm{T}$-fauna and $\mathrm{C}$ or $\mathrm{T}_{\text {final }}$, which is also surprising since the amount of added "higher quality" animal organic matter was high in this treatment (YEE \& JULIANO 2012). Different hypotheses could explain this result: first, the experiment was carried out for 14 days, which could be too long to witness such short-term effects of an animal pulse. Indeed, during these two weeks, the added organisms' density might have been levelled by predation and/or by behavioural migration resulting from the potentially increased competition for space and resources inside the mesocosms. Secondly, since it was very difficult to really estimate the invertebrates' density inside the added litter without over disturbing the community, it could be that the density of added macrofauna was too low, inducing damped responses within the first days of the experiment.

Except for G. fucicola, all the other species experienced a significant increase in density in both T-fauna and T-defaun. The most striking observation is the seven-fold increase in density of G. aequicauda. It must be noted that the SIMPER analysis coupled to the n-MDS ordination confirmed that the increased / reduced densities of these 7 species allow the strong differentiation of the controls and the two treatments into two very distinct groups, the first being what could be expected from a "natural" community at that moment of the year, and the second potentially reacting to a resource pulse. The significant density increase of detritivores is potentially a mixed result of the active colonisation of the treatments' mesocosms by new G. aequicauda and of increased juvenile recruitment. While G. aequicauda is a very active swimmer (swimming speed up to $20 \mathrm{~cm} \cdot \mathrm{s}^{-1}$ in laboratory conditions) with a moving capacity considered relatively high for a benthic amphipod (VERSCHUT et al. 2015; LONGO et al. 2016), amphipod juveniles are a major component of litter meiofauna (MASCART et al. 2015a) and the experiment duration (14 days) would have allowed juveniles to reach the $500 \mu \mathrm{m}$ size needed to be retained by sieving.

The drastically increased density of a purely detritivore species such as G. aequicauda could be a credible explanation for the absence of difference in litter biomass between the controls and the treatments. Even if loss of material is never excluded, the presence of these important litter fragmenters and consumers associated with potentially lower oxygen concentrations and increased microbial activity could explain the increased degradation of litter in the treatments.

Gammarus aequicauda is known to be a detritivore, ingesting and assimilating P. oceanica dead leaves (LEPOINT et al. 2006; MiCHEL et al. 2015), biting but also shredding dead seagrass leaves (MANCINELLI 2012). It is a species found in various macrophytodetritic accumulations such as Phragmites accumulations (MANCINELLI et al. 2009) or Cymodocea/Zostera accumulations (MANCINELLI \& ROSSI 2002). The density increase of the most important detritivore species found in the accumulation of 
REMY F. et al., Effect of resource pulse on seagrass macrophytodetritus fauna

dead litter exported P. oceanica constitutes to one of the main observations of this study. Indeed, such detritivore-based responses to resource pulses are mostly found in terrestrial ecosystems (NowLIN et al. 2008; YANG et al. 2008), but are also found in other aquatic phanerogam detritus accumulations such as reed (MANCINELli et al. 2005). The exported dead $P$. oceanica litter accumulations present similarities with terrestrial forest ecosystems from the pulse point of view. Like most terrestrial plants, $P$. oceanica is a flower plant which sheds its leaves in autumn. Like most terrestrial macrophyte-driven ecosystems, these leaves form an important detrital pool with associated well-developed "detrital" food webs. These characteristics lead to a bottom-up response to pulses, typical of some forest ecosystems: detritivore organisms are able to take much more advantage of resource pulses (YANG 2006) than herbivores, which is what was observed during this experimental study. Indeed, G. fucicola, the most dominant species in natural accumulations, is considered as a detritivore/herbivore species, probably relying more on epiphytes than G. aequicauda (LEPOINT et al., 2006 MiCHEL et al. 2015). Moreover, some observations indicate that $G$. fucicola could need more degraded litter (more colonised by microbes) than G. aequicauda (Compère \& Trevisan, unpublished data). In our control litter, G. fucicola dominated the assemblage as it usually does the natural litter, but litter added for our treatment was "new" litter (senescing leaves) coming directly from the meadow, probably in the first stage of the degradation process. Therefore, the two gammarids do potentially not share the same detritivore niche: G. aequicauda could be regarded as a pioneer detritivore and G. fucicola as a secondary coloniser.

Such primary bottom-up responses are not the only responses to pulses. Top-down responses of predators always follow after a certain lag time (secondary response) (OSTFELD \& KEESING 2000; CHESSON et al. 2004; YANG et al. 2010), depending on the generation time of the organisms comprising the impacted community. This study was not primarily designed to document such longer time-sensitive outcomes and secondary responses but the observed significant abundance increase of carnivore species such as Palaemon xiphias and Athanas nitescens could be linked to the fresh litter input. This secondary response was relatively fast and occurred in less than 14 days, which probably also implies an active colonisation of the treatment mesocosms by these two species. This response could be congruent with HOLT (2008), who stated that a very intense but very brief resource pulse could result in a drastic primary consumer density increase within the first day after the event, and a moderate but fast predator density increase within the first 10 days after the event. This is also in accordance with Nowlin et al. (2008), who stated that invertebrate communities composed of organisms with short generation times could respond very quickly to resource pulses. The increase of $P$. xiphias and A. nitescens densities could thus be a result of the rapid increase of one of their potential prey, G. aequicauda. This predatory response could also induce cascading effects on other prey, like the very abundant G. fucicola. The important decrease of G. fucicola density could be the result of the potential reduced fitness of the species, of the increased competitiveness of G. aequicauda (emigration effect) but also of increased predatory stress due to $P$. xiphias or $A$. nitescens (predation effect).

Another result was the non-negligible presence of Nebalia strausi in both treatments, and its absence from the control and $\mathrm{T}_{\text {final }}$. Considering the very small size of $N$. strausi, active migration inside T-defaun. and T-fauna is more than probable. The Nebalia genus (OKEY 2003) and specifically the species $N$. strausi is known to be highly tolerant to hypoxia (GALLMETZER et al. 2005; REMY 2016), indicating that the addition of dead leaves to the treatment mesocosms potentially induced a certain level of hypoxia.

\section{Conclusions}

In conclusion, this experimental study provides insights into the impact of a resource pulse on the vagile macrofauna associated with an exported dead $P$. oceanica litter accumulation. We demonstrated that several major species of invertebrates showed drastic density variations in response to the pulse treatments, and that the driving parameter was the input of "fresh" litter and not the presence of the associated animal fraction. More precisely, the marked increase in G. aequicauda density, a pioneer detritivore species, was of major importance in our comprehension of the pulse response of the litter 
macrofauna community and how it could increase litter degradation and assimilation in the coastal macrofauna food web. Indeed, this response of a purely detritivore species is found in other terrestrial ecosystems but also in brackish areas and is a sign that exported $P$. oceanica litter accumulations and terrestrial macrophyte-driven ecosystems potentially share more common characteristics than we previously thought.

Because macrophytodetritic habitats (e.g., seagrass or reed for example) may be found everywhere in the shallow coastal areas of the Mediterranean Sea (MATEO \& ROMERO 1997; MANCINELLI et al. 2007; LEPOINT et al. 2006), they probably play an important role in local trophic webs (MOORE et al. 2004) and in the seagrass-based C cycle. However, in comparison with other detrital ecosystems, the impact of the natural dynamics (e.g., resource pulses) affecting these accumulations and their functioning (i.e., $\mathrm{C}$ cycling, nutrients cycling, biodiversity driver) remain largely understudied and our understanding of them would benefit from them receiving particular attention in future research.

\section{Acknowledgements}

The authors kindly thank the STARESO field station staff for their assistance during sampling setup, especially Arnaud Abadie for his assistance during sampling. The authors also warmly thank Renzo Biondo for his help during setup design. F. Remy acknowledges a F.R.I.A. grant (Belgian National Fund for Research training in Industry and in Agriculture). This study was conducted within the frame of FRS-FNRS research project FRFC 2.4511.09. G. Lepoint is a Research Associate of the FRS-FNRS. This is publication MARE number 342 .

\section{References}

Anderson M., Gorley R.N. \& Clarke R.K. (2008b). Permanova + for Primer: Guide to Software and Statistical Methods. Available from http://www.primer-e.com/permanova.htm [accessed 30 Jan. 2017].

ANDERSON W.B., WAit D.A. \& STAPP P. (2008a). Resources from another place and time: Responses to pulses in a spatially subsidized system. Ecology 89 (3): 660-670. https://doi.org/10.1890/07-0234.1

Anesio A., Abreu P. \& Biddanda B. (2003). The role of free and attached microorganisms in the decomposition of estuarine macrophyte detritus. Estuarine, Coastal and Shelf Science 56 (2): 197-201. https://doi.org/10.1016/S0272-7714(02)00152-X

Boudouresque C.F., Bernard G., Bonhomme P., Charbonnel E., Diviacco G., Meinesz A., Pergent G., Pergent-Martini C., Ruitton S. \& Tunesi L. (2006). Préservation et conservation des herbiers à Posidonia oceanica. Ramoge publications, Monaco: 1-200.

Chesson P., Gebauer R.L.E., Schwinning S., Huntly N., Wiegand K., Ernest M.S.K., Sher A., Novoplansky A. \& Weltzin J.F. (2004). Resource pulses, species interactions, and diversity maintenance in arid and semi-arid environments. Oecologia 141 (2): 236-253. https://doi.org/10.1007/ $\underline{\mathrm{s} 00442-004-1551-1}$

Clarke K.R. \& Gorleyr N. (2006). V6: User manual/tutorial. Primer-E Ltd., Plymouth: 93.

DimeCH M., BORG J.A. \& SCHEMBRI P.J. (2006). Motile macroinvertebrate assemblages associated with submerged Posidonia oceanica litter accumulations. Biologia Marina Mediterranea 13: 130-133.

Gallmetzer I., Pflugfelder B., Zekely J. \& Ott J.A. (2005). Macrofauna diversity in Posidonia oceanica detritus: distribution and diversity of mobile macrofauna in shallow sublittoral accumulations of Posidonia oceanica detritus. Marine Biology 147 (2): 517-523. https://doi.org/10.1007/s00227-005$\underline{1594-9}$

GILLER P.S. (1996). The diversity of soil communities, the 'poor man's tropical rainforest'. Biodiversity and Conservation 5 (2): 135-168. https://doi.org/10.1007/BF00055827 
REMY F. et al., Effect of resource pulse on seagrass macrophytodetritus fauna

GOBert S (2002). Variations spatiale et temporelle de l'herbier à Posidonia oceanica (L.) Delile. Université de Liège. PhD Thesis. University of Liège, Liège, 207pp.

HoLT R.D. (2008). Theoretical perspectives on resource pulses. Ecology 89: 671-681. https://doi. org/10.1890/07-0348.1

LAKE P.S. (2000). Disturbance, patchiness, and diversity in streams. Journal of the North American Benthological Society 19 (4): 573-592. https://doi.org/10.2307/1468118

LEMKE A.M., LEMKE M.J. \& BENKE A.C. (2007). Importance of detrital algae, bacteria, and organic matter to littoral microcrustacean growth and reproduction. Limnology and Oceanography 52: 21642176. https://doi.org/10.4319/1o.2007.52.5.2164

LePoint G., CoX A.-S., DAuby P., PouliceK M. \& GoberT S. (2006). Food sources of two detritivore amphipods associated with the seagrass Posidonia oceanica leaf litter. Marine Biology Research 2 (5): 355-365. https://doi.org/10.1080/17451000600962797

Leroux S.J. \& Loreau M. (2012). Dynamics of Reciprocal Pulsed Subsidies in Local and MetaEcosystems. Ecosystems 15 (1): 48-59. https://doi.org/10.1007/s10021-011-9492-0

Levi T., Kilpatrick A.M., Barfield M., Holt R.D., MAngel M. \& Wilmers C.C. (2015). Threshold levels of generalist predation determine consumer response to resource pulses. Oikos 124: 1436-1443. https://doi.org/10.1111/oik.01487

Longo E., Verschut T., CArrozzo L., Zotti M., Mancinelli G. (2016). Inter-and intra-specific variation in movement behaviour of benthic macroinvertebrates from a transitional habitat: a laboratory experiment. Rendiconti Lincei 27 (2): 281-290. https://doi.org/10.1007/s12210-015-0475-5

MANCINELLI G. (2012). To bite, or not to bite? A quantitative comparison of foraging strategies among three brackish crustaceans feeding on leaf litters. Estuarine, Coastal and Shelf Science 110: 125-133. https://doi.org/10.1016/j.ecss.2012.04.002

MANCINELLI G. \& Rossi L. (2002). The influence of allochthonous leaf detritus on the occurrence of crustacean detritivores in the soft-bottom macrobenthos of the Po River Delta Area (northwestern Adriatic Sea). Estuarine, Coastal and Shelf Science 54 (5): 849-861. https://doi.org/10.1006/ecss.2001.0861

MANCINELli G., SABETTA L. \& BASSET A. (2005). Short-term patch dynamics of macroinvertebrate colonization on decaying reed detritus in a Mediterranean lagoon (Lake Alimini Grande, Apulia, SE Italy). Marine Biology 148 (2): 271-283. https://doi.org/10.1007/s00227-005-0091-5

MANCINELli G., SABETTA L. \& BASSET A. (2007). Colonization of ephemeral detrital patches by vagile macroinvertebrates in a brackish lake: a body size-related process? Oecologia 151 (2): 292-302. https:// doi.org/10.1007/s00442-006-0586-x

Mancinelli G., Vignes F., SAngiorgio F., Mastrolia A. \& Basset A. (2009). On the potential contribution of microfungi to the decomposition of reed leaf detritus in a coastal lagoon: A laboratory and field experiment. International Review of Hydrobiology 94 (4): 419-435. https://doi.org/10.1002/ $\underline{\text { iroh.200811164 }}$

Mascart T., Lepoint G., Deschoemaeker S., Binard M., Remy F. \& De Troch M. (2015a). Seasonal variability of meiofauna, especially harpacticoid copepods, in Posidonia oceanica macrophytodetritus accumulations. Journal of Sea Research 95: 149-160. https://doi.org/10.1016/j.seares.2014.07.009

MAscart T., Agusto L., Lepoint G., Remy F. \& De Troch M. (2015b). How do harpacticoid copepods colonize detrital seagrass leaves? Marine Biology 162 (5): 929-943. https://doi.org/10.1007/s00227$\underline{015-2632-x}$ 
Mateo M.A. \& Romero J. (1997). Detritus dynamics in the seagrass Posidonia oceanica: Elements for an ecosystem carbon and nutrient budget. Marine Ecology Progress Series 151: 43-53. https://doi. org/10.3354/meps 151043

MATEO M.Á, SÁNCHEZ-LiZASo J-L, ROMERo J (2003). Posidonia oceanica 'banquettes': a preliminary assessment of the relevance for meadow carbon and nutrients budget. Estuarines Coastal Shelf Sciences 56: 85-90. https://doi.org/10.1016/S0272-7714(02)00123-3

Michel L, Dauby P., Gobert S., Graeve M., Nyssen F., Thelen N. \& Lepoint G. (2015). Dominant amphipods of Posidonia oceanica seagrass meadows display considerable trophic diversity. Marine Ecology 36 (4): 969-981. https://doi.org/10.1111/maec.12194

Mölter T., Schindler D., Albrecht A.T. \& Kohne U. (2016). Review on the projections of future storminess over the North Atlantic European Region. Atmosphere 7 (4): 60. https://doi.org/10.3390/ $\underline{\operatorname{atmos} 7040060}$

Moore J.C., Berlow E.L., Coleman D.C., Ruiter P.C., Dong Q., Hastings A., Johnson N.C., McCann K.S., Melville K., Morin P.J., Nadelhoffer K., Rosemond A.D., Post D.M., Sabo J.L., SCOW K.M., VANNI M.J. \& WALL D.H. (2004). Detritus, trophic dynamics and biodiversity. Ecology Letters 7 (7): 584-600. https://doi.org/10.1111/j.1461-0248.2004.00606.x

Nowlin W.H., VANNI M.J. \& YANG L.H. (2008). Comparing resource pulses in aquatic and terrestrial ecosystems. Ecology 89 (3): 647-659. https://doi.org/10.1890/07-0303.1

OKEY T.A. (2003). Macrobenthic colonist guilds and renegades in Monterey Canyon (USA) drift algae: Partitioning multidimensions. Ecological Monographs 73 (3): 415-440. https://doi.org/10.1890/01$\underline{4088}$

Ostfeld R.S. \& KeEsing F. (2000). Pulsed resources and community dynamics of consumers in terrestrial ecosystems. Trends in Ecology \& Evolution 15 (6): 232-237. https://doi.org/10.1016/S0169$\underline{5347(00) 01862-0}$

Pasqualini V., Pergent-Martini C., Clabaut P. \& Pergent G. (1998). Mapping of Posidonia oceanica using Aerial Photographs and Side Scan Sonar: Application off the Island of Corsica (France). Estuarine, Coastal and Shelf Science 47 (3): 359-367. https://doi.org/10.1006/ecss.1998.0361

REMY F. (2016) Characterization, dynamics and trophic ecology of macrofauna associated to seagrass macrophytodetritus accumulations (Calvi Bay, Mediterranean Sea). PhD Thesis. University of Liège, Liège, $285 \mathrm{pp}$.

Romero J., Pergent G., Pergent-Martini C., Mateo M.A. \& Regnier C. (1992). The detritic compartment in a Posidonia oceanica meadow: litter features, decomposition rates, and mineral stocks. PSZNI Marine Ecology 13: 69-83. https://doi.org/10.1111/j.1439-0485.1992.tb00341.x

Sturaro N., Caut S., Gobert S., Bouquegneau J.-M. \& Lepoint G. (2010). Trophic diversity of idoteids (Crustacea, Isopoda) inhabiting the Posidonia oceanica litter. Marine Biology 157 (2): 237247. https://doi.org/10.1007/s00227-009-1311-1

Sturaro N., Lepoint G., Pérez-Perera A., Vermeulen S., Pieraugusto P., Navone A. \& Gobert S. (2014). Seagrass amphipod assemblages in a Mediterranean marine protected area: a multiscale approach. Marine Ecology Progress Serie 506: 175-192. https://doi.org/10.3354/meps10776

VALENTINE J.F. \& HeCK K.L. (1999). Seagrass herbivory: evidence for the continued grazing of marine grasses. Marine Ecology Progress Series 176: 291-302. https://doi.org/10.3354/meps176291

VERSCHUT T.A., MEINERI E. \& BASSET A. (2015). Biotic interactions affect the colonization behavior of aquatic detritivorous macroinvertebrates in a heterogeneous environment. Estuarine, Coastal and Shelf Science 157: 120-128. https://doi.org/10.1016/j.ecss.2015.03.014 
YANG L.H. (2006). Interactions between a detrital resource pulse and a detritivore community. Oecologia 147: 522-532. https://doi.org/10.1007/s00442-005-0276-0

Yang L.H., Bastow J.L., Spence K.O. \& Wright A.N. (2008). What can we learn from resource pulses? Ecology 89 (3): 621-634. https://doi.org/10.1890/07-0175.1

YAng L.H., Edwards K.F., Byrnes J.E., BAStow J.L., Wright A.N. \& SPEnCE K.O. (2010). A metaanalysis of resource pulse-consumer interactions. Ecological Monographs 80 (1): 125-151. https://doi. org/10.1890/08-1996.1

YeE D.A. \& JULiAno S.A. (2012). Concurrent effects of resource pulse amount, type, and frequency on community and population properties of consumers in detritus-based systems. Oecologia 169 (2): 511-522. https://doi.org/10.1007/s00442-011-2209-4

Manuscript received: 8 June 2016

Manuscript accepted: 3 November 2016

Published on: 14 July 2017

Branch editor: Marleen De Troch 